\title{
BISTUA
}

Revista de la Facultad de Ciencias Básicas

ISSN: 0120-4211 - ISSN-e: 2711-3027

\section{Estudio por simulación de un radio de acción de la posible explosión de una caldera, mediante el uso de un material explosivo}

\author{
Study by simulation of a radius of action of the possible explosion of a boiler, through the use of an explosive material
}

\author{
Mayerly García a ; Johan Ávila a; John Rincón a; Jorge Fonseca a \\ ${ }^{a}$ Escuela de Ingenieros Militares, Colombia.
}

\section{Resumen}

En Colombia desde hace muchos años por cuestiones de conflicto interno político, los explosivos han sido vistos más como una amenaza y no como una herramienta, razón por la cual en el imaginario del común las personas al escuchar la palabra "explosivo" lo asimilan más con algún tipo de actividad terrorista. Por tal razón este trabajo busca mostrar que, los explosivos a través de un uso profesional y responsable son una herramienta eficiente y eficaz para la mitigación de fallas o posibles accidentes en los procesos de producción industrial.

El artículo presenta el uso de material explosivo ANFO, como instrumento, para la predicción de daños en estructuras aledañas o infraestructura en caso de explosión de una caldera; empleada en los procesos o líneas de producción de vapor de la Embotelladora de Coca Cola, FEMSA en la ciudad de Bogotá. Finalmente, los resultados obtenidos fueron hechos mediante la elaboración de un modelo a escala de una caldera, comparación de características técnicas de operación de caldera en relación con el explosivo ANFO dadas por el fabricante; y medición de vibraciones, determinando la estimación de velocidad pico de partícula.

Palabras clave: Caldera; explosión; ANFO; sobrepresión; destrucción.

\section{Abstract}

In Colombia for many years due to internal political conflict issues, explosives have been seen more as a threat and not as a tool, which is why in the common imagination, when people hear the word "explosive" they assimilate it more with some type of terrorist activity. For this reason, this work seeks to show that explosives through professional and responsible use are an efficient and effective tool for mitigating failures or possible accidents in industrial production processes.

The paper presents the use of explosive material ANFO, as an instrument, for the prediction of damage to surrounding structures or infrastructure in the event of a boiler explosion; used in the processes or steam production lines of the Coca Cola Bottling Company, FEMSA in the city of Bogota. Finally, the results obtained were made by means of the elaboration of a scale model of a boiler, comparison of the technical characteristics of the boiler operation in relation to the explosive ANFO given by the manufacturer; and vibration measurement, determining the particle peak velocity estimate.

Keywords: Boiler; explosion; ANFO; overpressure; destruction.

\section{Introducción}

La empresa Coca Cola- FEMSA, mediante un estudio de seguridad industrial con sus profesionales de operación determino que el principal riesgo de seguridad industrial es la explosión de alguna de sus calderas por causas diversas. Por tal razón se plantea como objetivo mitigar los posibles daños de dichas fuentes de peligro; propiciando dicha posibilidad el siguiente interrogante de investigación. ¿Cómo determinar el radio de acción de la explosión de una de las calderas de la planta Coca Cola FEMSA, por medio de la simulación a escala realizada con la detonación de un material explosivo?
La investigación plantea la posible explosión de una o las tres calderas de la planta de Coca Cola FEMSA, teniendo como objetivo general: a) la realización y comparación entre una explosión balística de una caldera de la planta embotelladora de Coca Cola FEMSA mediante un material explosivo (agente de Voladura "ANFO"), b) un modelo de caldera a escala reducida, para lo cual se recreara la detonación de un modelo a escala de una de las calderas reales utilizando un explosivo tipo ANFO.

Lo anterior, se asemejara a una explosión mecánica de la real; pudiendo así tomar datos mediante la utilización del equipo Sismógrafo y geófono Mini-Seis III, medidas de cráter, distancias de restos, y toda la información posible a recolectar como resultado de la detonación controlada. De 
hecho, para evaluar el rango de presión de trabajo de la caldera y así simular su explosión, con la detonación del explosivo. Además, del modelo a escala se requiere determinar teóricamente el volumen de gases y otras variables de operación como la presión de la caldera. Finalmente, comparar la presión de detonación y volumen teórico de gases que se produce con el material explosivo.

Esta información, inicialmente es un insumo para analizar la posible catástrofe, escalar los resultados y demostrar los alcances. Igualmente, sugerir así un material resistente que permita mitigar la afectación producto de la explosión de la caldera, partiendo de las especificaciones técnicas ofrecidas por el comercio de los materiales, partes y repuestos que se pueden encontrar en todo el mercado.

La investigación aplica un enfoque de la búsqueda de una solución; a un problema del sector productivo colombiano.

\section{Método}

La investigación comprende aspectos teóricos y conceptuales que se implementaron en el desarrollo de las actividades. Inicialmente, los conceptos importantes de definición de caldera y explosivos para así obtener una idea del alcance y explicar las características del ANFO; como material explosivo a utilizar en la práctica. Por otra parte, se define que es una explosión, las posibles causas a generar y el tipo de investigación. Finalmente, se continúa con los efectos producidos por este tipo de explosión y las vibraciones generadas.

Una caldera de vapor es un equipo térmico cuya función es utilizar la energía calorífica de un combustible, para generar vapor de agua, en diferentes condiciones utilizables en procesos industriales.

En términos simples se considera que una caldera, es básicamente un recipiente a presión, cerrado, en el cual se calienta agua para uso externo del mismo por aplicación directa de calor resultado de la combustión de un combustible (solido, líquido o gaseoso). Este fluido puede ser calentado hasta llegar a evaporarse en el caso que se requiera vapor de agua.

Se denomina explosivo a toda sustancia química o compuesto la cual tenga la capacidad de transformarse por medio de estímulos de presión o calor, en productos condensados y gaseosos. El volumen inicial ocupado por el explosivo se convierte en una masa gaseosa que alcanza elevadas temperaturas y por ende altas presiones [1].

El ANFO [2] es un agente de voladura conformado por la mezcla química de nitrato de amonio, biodiesel o mezclas de hidrocarburos, el cual es sensible a la iniciación por un multiplicador - Pentofex, el ANFO tiene una baja resistencia a la humedad (higroscópico) por lo cual no es apropiado para operaciones subterráneas. Es muy seguro durante su manipulación y uso. Permite, ser cargado en forma manual o neumática en barrenos, pudiéndose utilizar al detal o encartuchado. Es empleado en voladuras a cielo abierto tales como: minería o explotación de rocas blandas o semiduras en canteras y en obras civiles. Se utiliza principalmente como explosivo de carga de columna [3].

Una explosión es el escape comprimido de gases el cual está acompañado de altas temperaturas, violentas sacudidas y ruidos estrepitosos. Al momento de la explosión se produce gran cantidad de gases los cuales se dilatan rápidamente, comprimiendo el aire y formando una onda explosiva. La presión del gas se mueve hacia fuera como un fuerte viento detrás del frente de la onda explosiva llamado "frente de choque".

Cuando se produce una explosión, se forman nubes de gases bastante comprimidos que alcanzan altas temperaturas y generan presiones de alrededor de 700 toneladas por pulgada cuadrada $(635.040 \mathrm{Kg} / \mathrm{cm} 2)$ en la atmósfera que rodea el punto de la explosión. Estos gases se propagan hacia la parte externa de la explosión.

La onda de presión explosiva se propaga hacia la parte externa de la detonación en forma esférica como la ola de un maremoto gigante, golpeando y destrozando todo objeto que encuentra en su camino. A mayor distancia recorrida por la onda de presión desde el punto de detonación, tanto mayor es su desaceleración hasta que llega a convertirse en una onda sonora y luego a disiparse en el ambiente. Esta onda se llama "onda de presión explosiva" y forma lo que se conoce como "frente de choque" [4].

El tipo de explosión que se produce es mecánica la cual consiste en la conversión gradual de una sustancia, como el agua, en gas o vapor. La presión creciente del vapor dentro de un recipiente sobrepasara la resistencia estructural del mismo generando la explosión con alta liberación de energía.

La embotelladora de Coca Cola FEMSA, luego del análisis de sus profesionales de operación, considero que alguna de sus 3 calderas tiene el riesgo explotar por causas diversas; por ende, esta explosión generaría grandes daños. En virtud de esta situación, ellos han tratado de mitigar los posibles daños realizando el análisis de riesgos y su posible consecuencia $[5,6]$. Para ello se desarrolla esta investigación buscando conocer la mayor presión de trabajo de la caldera y así establecer un posible radio de afectación producto de la explosión de una de las tres calderas utilizadas en la fábrica. La investigación se realizó mediante la simulación de la detonación controlada de un modelo a escala reducida de la caldera (1:49) de $700 \mathrm{hp} \mathrm{la}$ cual es la caldera de mayor 
tamaño capacidad que se encuentra en la embotelladora. (Fig.1)

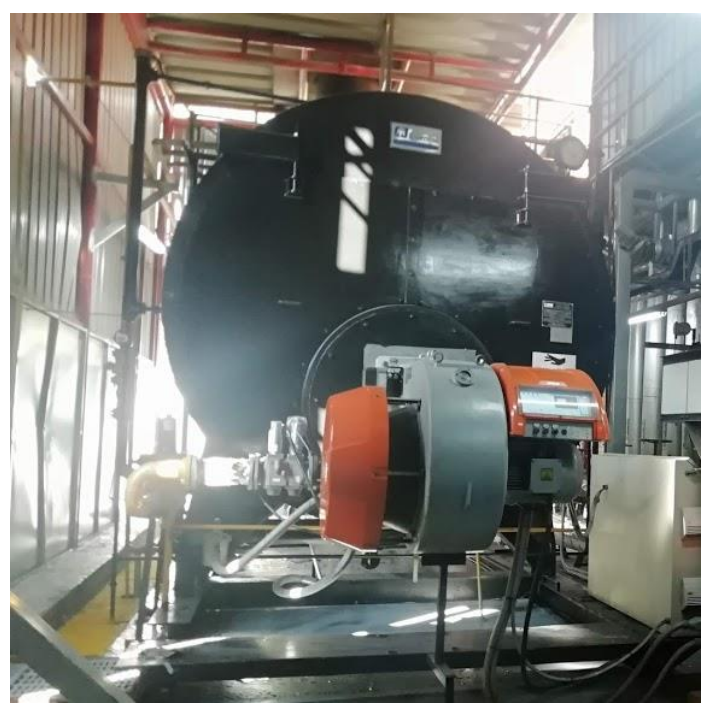

Figura 1 caldera de $700 \mathrm{HP}$ - Planta de coca cola Fuente: Autor(es)

El modelo a escala tiene una altura de $17 \mathrm{~cm}$, ancho de $17 \mathrm{~cm}$ y largo de $50 \mathrm{~cm}$, Fig. 2.

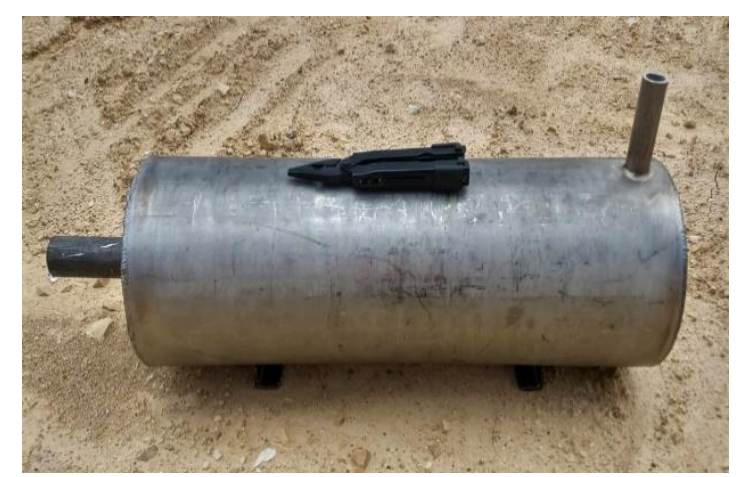

Figura 2. Modelo a escala 1:49 de la caldera de $700 \mathrm{Hp}$. Fuente: Autor(es)

El material utilizado para la fabricación del modelo a escala fue:

- $\quad$ Cuerpo y placas: SA 516 grado 70.

- Tubería: SA 178 (con costura) o 192 (sin costura).

- Bases y soporte: Acero estructural A36.

- Aislamiento interno: Fibra de vidrio y manta cerámica.

La detonación controlada del modelo a escala se efectuó con todos los parámetros de seguridad utilizando los siguientes materiales (Fig.3):

- $\quad$ ANFO (Agente de voladura).

- Cordón detonante de 3 gramos.
- $\quad$ Detonador fuerza $N^{\circ} 8$.

- Mecha de seguridad.

- Sismógrafo - Geófono

- Caldera a escala reducida.

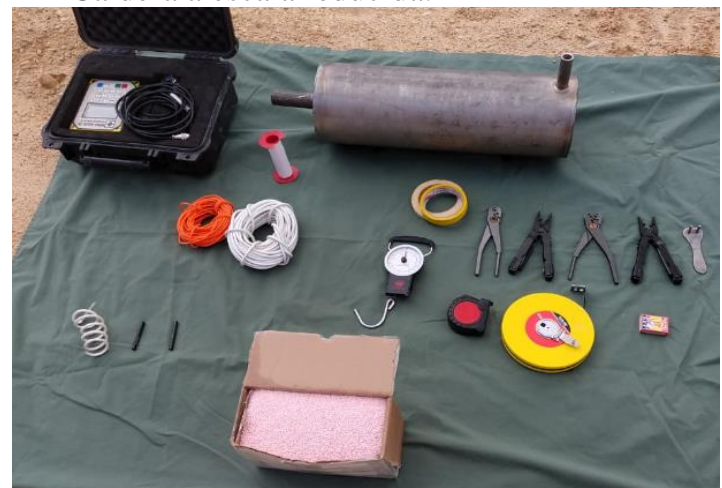

Figura 3. Materiales, equipos y herramientas utilizados en la detonación. Fuente: Autor(es)

Por último se empleó un sismógrafo (Fig.4), el cual es un instrumento utilizado para medir hasta los más mínimos movimientos de la Tierra dependiendo del tipo de equipo y la instalación de los geófonos. El instrumento está conformado por un sensor llamado sismómetro con capacidad de detectar el movimiento terrestre, recolectando los datos que recoge a un sistema de registro. Dicha información obtenida por este equipo proporciona una visión general del comportamiento terrestre que afectó la detonación controlada de la caldera hecha a escala.

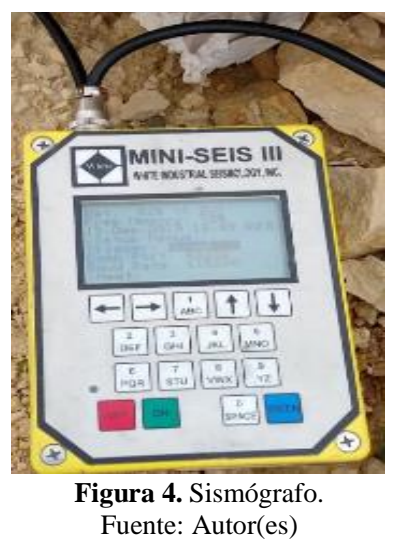

Finalmente, en el capítulo de resultados, se realizan los cálculos comparativos para determinar la cantidad de ANFO a utilizar en la caldera a escala. Los datos de la caldera a tamaño real se hicieron por medición de la caldera y uso de la ficha técnica del fabricante [7].

Mediante, el empleo de la ecuación de los gases ideales a condiciones normales $\left(273^{\circ} \mathrm{K}\right.$ y 1 atm de presión) se calcula la cantidad del volumen de gases producidos por la detonación del ANFO [2]. Por último, se estima la cantidad requerida de ANFO modelo a escala de caldera y las vibraciones, con el volumen de gases producidos por la 
explosión del agente de voladura en relación con el volumen vapor de agua de la caldera como gas ideal a condiciones normales, 27700 libras; simulando una detonación de la misma magnitud para la detonación de la caldera de $700 \mathrm{Hp}$.

\section{Resultados}

La investigación se desarrolló por medio de expresiones matemáticas $\operatorname{Ec(1)-(7);~enfocándose~principalmente~en~las~}$ Ec(5)-(7). Es decir, en el volumen o energía de burbuja, gases del ANFO, y la capacidad de la caldera para producir vapor de agua, considerando inicialmente el vapor de agua como gas ideal. Esto es, 27700 libras de vapor de agua en condiciones normales de operación. Las expresiones matemáticas adicionales de presión de detonación y volumen de gases de la caldera a escala real $(700 \mathrm{Hp})$ [7], se determinaron con el fin de comparar y correlacionar la viabilidad de simulación, con las características técnicas de presión de detonación y volumen de gases del agente explosivo de voladura ANFO [2].

Finalmente, para el ANFO y caldera, los parámetros de presión de detonación, se excluyen por ser energía de choque y no energía de burbuja [4]. También, el volumen del agua en la caldera por ser fase liquida y no vapor de agua para ser considerado como un gas.

La presión de detonación de la caldera es de 36225 Psi. Valor límite de presión la caldera en caso de generar una explosión mecánica, venciendo en dicho valor de presión la resistencia del material de fabricación de la caldera [7].

Con estos datos se estimó la cantidad de explosivo y el modelo de caldera a escala reducida a construir; siendo la cantidad de $1,058 \boldsymbol{K g}$ ANFO a utilizar en la detonación controlada.

- $\quad$ Presión de detonación la caldera: $\quad 36.225$ Psi

$$
\begin{array}{cr}
0,00689476 \mathrm{MPa} & 1 \mathrm{PSI} \\
X & 36.225 \mathrm{Ps} \\
& \\
& \boldsymbol{X}=249,59 \mathrm{Mpa}
\end{array}
$$

- Presión de detonación del ANFO

$$
\boldsymbol{P D}=\frac{10^{-3}}{4} * \rho_{E x p} * V O D^{2}
$$

Dónde:

$\boldsymbol{\rho}_{\text {Exp }}$ : Densidad del ANFO

VOD : Velocidad de detonación

$$
\begin{gathered}
\boldsymbol{P D}=\frac{10^{-3}}{4} * 0,85^{\mathrm{gr}} / \mathrm{cm}^{3} *(3000 \mathrm{~m} / \mathrm{s})^{2} \\
\boldsymbol{P D}=\mathbf{1 . 9 1 2 , 5} \mathbf{M P a}
\end{gathered}
$$

- Dimensiones de la caldera

$$
\begin{aligned}
& \boldsymbol{L}=762 \mathrm{~cm}+25,4 \mathrm{~cm}=\mathbf{7 8 7}, \mathbf{4} \mathrm{cm} \\
& \emptyset=243,2 \mathrm{~cm}+12,7 \mathrm{~cm}=255,9 \mathrm{~cm} \\
& A=\frac{\pi}{4} * \emptyset^{2} \\
& \boldsymbol{A}=\frac{\pi}{4} * 255,9^{2} \mathrm{~cm} \\
& \boldsymbol{A}=\frac{\pi}{4} *(255,9 \mathrm{~cm})^{2} \\
& A=51.431,65 \mathrm{~cm}^{2}
\end{aligned}
$$

- Volumen total de la caldera

$$
\begin{gathered}
\mathbf{V}=\mathbf{A} * \mathbf{L} \\
\boldsymbol{V}_{\text {total }}=51.431,65 \mathrm{~cm}^{2} * 787,4 \mathrm{~cm} \\
\boldsymbol{V}_{\text {total }}=40 \times 497.281,21 \mathrm{~cm}^{3} \\
\boldsymbol{V}_{\text {total }}=\mathbf{4 0 . 4 9 7 , 2 8 ~} \mathbf{L T}
\end{gathered}
$$

- Volumen vapor de agua de la cadera (condiciones normales): $27.700 \mathrm{Lb}$.

$$
\begin{aligned}
& \text { 453,6 gr } \quad 1 \mathrm{Lb} \\
& \text { X } \quad 27.700 \mathrm{Lb} \\
& \boldsymbol{X}=12 ‘ 564.720 \mathrm{gr} \\
& \boldsymbol{V}_{\text {H2o CAL }}=\frac{12^{‘} 564.720 \mathrm{gr}}{1^{g x} / \mathrm{cm}^{3}} \\
& \boldsymbol{V}_{\text {H2O CAL }}=12^{‘} 564.720 \mathrm{~cm}^{3} \\
& V_{H 2 O C A L}=12.564,72 \mathrm{LT}
\end{aligned}
$$

- Estimación volumen de gases ideales para el ANFO

Se estima el volumen de gases producidos por el ANFO a partir de la ecuación de gases ideales en condiciones normales $\left(273{ }^{\circ} \mathrm{K}\right.$ y 1 atm de presión) cuyo valor es 22,4 litros $[4,8]$.

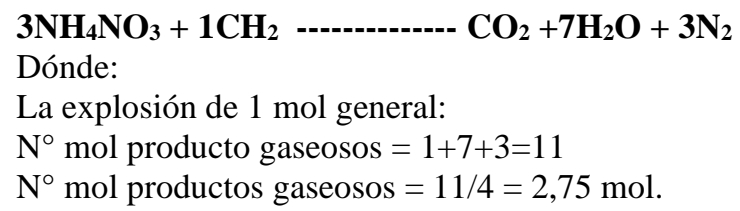


Los productos de gases a $273^{\circ} \mathrm{K}$ y a presión de 1 atmosfera es de:

$$
\begin{gathered}
2,75 \mathrm{~mol} * 22,4 \mathrm{LT} / \mathrm{mol} \\
\text { VOL } \text { Explosion ANFO }_{\text {An }}=61,6 \mathrm{LT}
\end{gathered}
$$

- $\quad$ Relación cantidad equivalente de ANFO para generar la explosión aproximada como la generada por sobre presión de la caldera

$$
\begin{array}{r}
\boldsymbol{C}_{\text {ANFO }}=\frac{V_{\text {vapor } \mathrm{H} 20 \mathrm{CAL}}}{V O L_{\text {Explosion } A N F O}} \\
\boldsymbol{C}_{\text {ANFO }}=\frac{12.564 .72 \mathrm{LT}}{61,6 \mathrm{LT}} \\
\boldsymbol{C}_{\text {ANFO }}=203,97 \text { veces }
\end{array}
$$

$$
\boldsymbol{C}_{\text {ANFo }}=203,97 * 254,3 \operatorname{gramos}(\text { peso molecular } A N F O)
$$

$$
C_{\text {ANFO }}=51869,571 \mathrm{gr}
$$

- Cantidad de ANFO para modelo a escala

El modelo está construido con una escala 1:49 (Fig.2)

$$
\begin{aligned}
& \mathbf{C}_{\mathrm{ANFO}}=\frac{\mathrm{C}_{\mathrm{ANFO}}}{E S C_{\text {modelo }}} \\
& \mathbf{C}_{\mathrm{ANFO}}=\frac{51869,571 \mathrm{gr}}{49} \\
& \mathbf{C}_{\mathrm{ANFO}}=1058,56 \mathrm{gr} \\
& \mathbf{C}_{\mathrm{ANFO}}=\mathbf{1 , 0 5 8} \mathbf{~} \mathbf{g}
\end{aligned}
$$

Finalmente, se realiza la toma de los datos de la presión del aire (Fig.5) a través del geófono (sismógrafo) obteniéndose los resultados de velocidad de pico de partícula (PPV) y frecuencia en el modelo a escala reducida de la caldera.

\section{Peaks and Frequencies \\ PPV Maximum: $38.2 \mathrm{~mm} / \mathrm{s}$ R: $30.0 \mathrm{~mm} / \mathrm{s} @ 204.8 \mathrm{~Hz}(0.0115 \mathrm{~s})$ \\ T: $11.5 \mathrm{~mm} / \mathrm{s} @ 170.7 \mathrm{~Hz}$ (0.00977 s)}

Figura 5. Registro Sismógrafo $\mathrm{N}^{\circ}$ 0286, Detonación Modelo a Escala. Lectura del sismógrafo. Fuente: Autores

Los resultados obtenidos por el sismógrafo muestran altas velocidades de pico de partícula, producto de la propagación de las ondas, en los tres componentes radial, transversal y vertical. Los mayores valores obtenidos en la detonación del ANFO en velocidad de pico partícula fueron de $38,2 \mathrm{~mm} / \mathrm{s}$ y de frecuencia a un valor de $204,8 \mathrm{~Hz}$. El sismógrafo fue ubicado a una distancia de $8,2 \mathrm{~m}$.
Estos datos fueron graficados por el equipo y al ser descargados mostro los picos causados por la detonación del ANFO en el modelo de caldera a escala reducida (Fig.6).

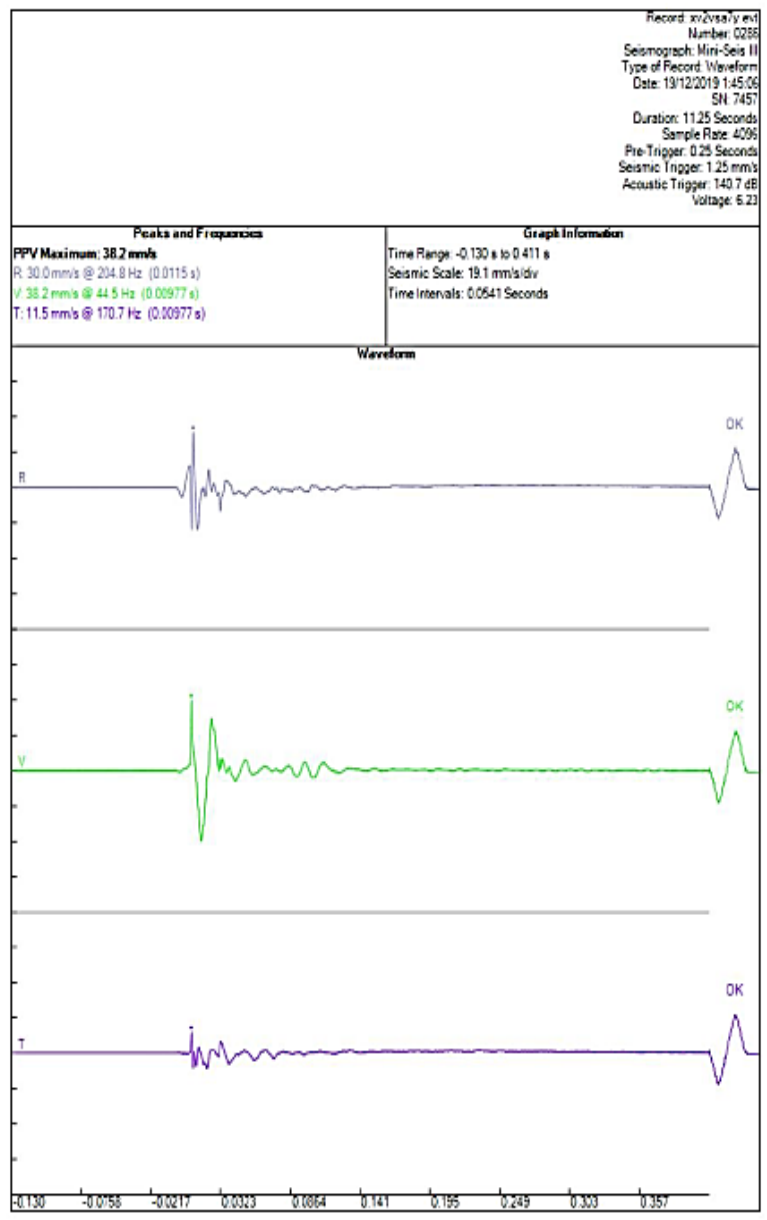

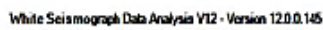

Figura 6. Lectura de la velocidad pico partícula (Registro del sismógrafo) Fuente: Lectura del sismógrafo.

El análisis de los datos obtenidos por el sismógrafo se realizó mediante el software AT-BLAST, el cual entrega una tabla y grafica de la presión de detonación que se produjo durante la detonación. De igual modo, entrega información mediante el análisis de la envolvente de afectación.

La Fig. 7 presenta la envolvente de afectación con 3 distancias estándar; cuyas presiones de detonación está en múltiplos de 7 . 


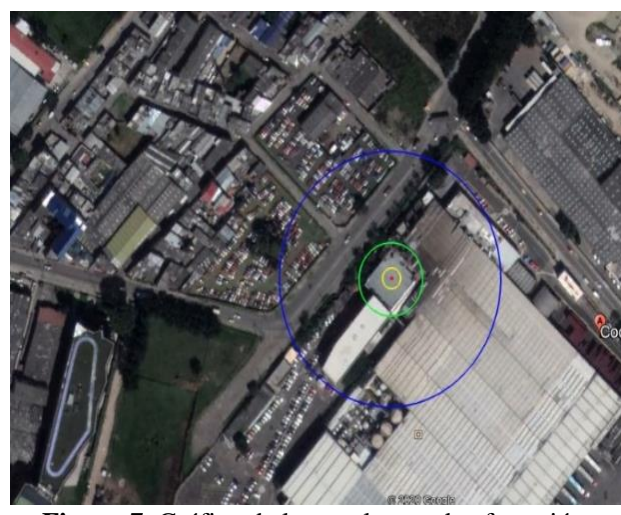

Figura 7. Gráfico de la envolvente de afectación. Fuente: Autores

La máxima presión de detonación obtenida por el software AT-BLAST fue de 70,95 Mpa con una distancia de afectación de la envolvente de 0,5 metros. (Tab. 1)

\begin{tabular}{|c|c|c|}
\hline Tabla 1. Datos de las envolventes con distancias estándar. \\
\hline CODIGO DE COLOR & $\begin{array}{c}\text { PRESION } \\
\text { Mpa }\end{array}$ & $\begin{array}{c}\text { DISTANCIA } \\
\text { metros }\end{array}$ \\
\hline & 71,0 & 0,5 \\
\hline & 0,700 & 5,8 \\
\hline & 0,070 & 22 \\
\hline & 0,007 & 75 \\
\hline
\end{tabular}

Fuente: Autores

Por otra parte, la información de la Tab 2, presenta la cantidad de energía (kilogramos de ANFO) equivalente de la caldera de $700 \mathrm{hp}$; al igual la distancia de afectación por parte de la envolvente en metros, junto con la velocidad de pico de partícula.

\begin{tabular}{c|c|c|c}
\multicolumn{4}{c}{ Tabla 2. Distancias de la Envolvente. } \\
\hline $\begin{array}{c}\text { PRESION } \\
\text { Mpa }\end{array}$ & $\begin{array}{c}\text { DISTANCIA } \\
\boldsymbol{m}\end{array}$ & $\begin{array}{c}\text { Peso } \\
\boldsymbol{A N F O} \\
\text { Kg }\end{array}$ & $\begin{array}{c}\text { Velocidad de } \\
\text { pico de } \\
\text { particula } \text { VPP } \\
\text { mm/seg }\end{array}$ \\
\hline 71,0 & 0,5 & 51,87 & 91374,03 \\
\hline 0,700 & 6,2 & 51,87 & 1432,34 \\
\hline 0,070 & 22 & 51,87 & 190,97 \\
\hline 0,007 & 75 & 51,87 & 26,84 \\
\hline \multicolumn{4}{|c|}{ Fuente: Autores }
\end{tabular}

De acuerdo a la norma alemana de vibraciones DIN 4150: 2000 valores de pico de partícula (VPP) a $40 \mathrm{~mm} / \mathrm{seg}$ en cualquier frecuencia afectan oficinas, edificaciones industriales con diseños robustos [9]. Por otra parte, situación similar es dada por el manual del especialista en voladuras Tabla 38.2 Pag. 565. [3].

Lo anterior permite deducir que la detonación de una caldera puede producir daños principalmente en la infraestructura y parte estructural de las edificaciones cercanas y en los sitios alrededor a la embotelladora Coca Cola FEMSA.

\section{Discusión Resultados}

De los datos obtenidos con el equipo de medición, se evidencia un aumento acelerado en las ondas por la gran cantidad de gases producidos durante a detonación.

Los daños producidos por la onda de Sobrepresión en las estructuras generan un radio de afectación por propagación de onda aérea. Daños a edificaciones circundantes de la planta, distancias críticas donde se puede evidenciar los daños según la onda de sobrepresión.

Para las distancias menores a 6 metros en un radio de afectación la onda de sobrepresión genera demolición total a las edificaciones aledañas. Por otra parte, la mitigación del impacto que genera de explosión, se determina que a hasta los $75 \mathrm{~m}$ metros del lugar de la explosión se pueden generar daños a los vidrios en un $90 \%$ las edificaciones aledañas.

Igual análisis ocurre con los valores de velocidad de pico de partícula (VPP) según norma alemana DIN 4150:2000; afectando considerablemente a estructuras que estén ubicadas a una distancia de 22 metros.

Realizando una relación entre un sismo y la explosión de la caldera, se puede deducir que la explosión se asimila a un SISMO DE MAGNITUD 2,3 EN LA ESCALA DE RITCHER, la explosión de la caldera genera una energía equivalente en TNT a $21,79 \mathrm{~kg}$. Generando daños significativos a las estructuras dentro de su radio de la distancia de seguridad mínima la cual es de $75 \mathrm{~m}$.

De acuerdo a la norma alemana de vibraciones DIN 4150: 2000 valores de pico de partícula (VPP) a $40 \mathrm{~mm} / \mathrm{seg}$ en cualquier frecuencia afectan oficinas, edificaciones industriales con diseños robustos [9]. Por otra parte, situación similar es dada por el manual del especialista en voladuras Tabla 38.2 Pag. 565. [3].

Lo anterior permite deducir que la detonación de una caldera puede producir daños principalmente en la infraestructura y parte estructural de las edificaciones y en los sitios alrededores a la embotelladora Coca Cola FEMSA.

\section{Conclusiones}

Como conclusión de suma importancia, se puede establecer que el rango de presión liberado al momento de la explosión de la caldera real de $700 \mathrm{Hp}$ de la embotelladora de Coca Cola FEMSA en la ciudad de Bogotá D.C. es alto; permitiendo la no cuantificación precisa de daño a una distancia menor de 75 metros. De acuerdo con las normas internacionales, el parámetro decisivo para medir el daño por vibraciones no solo en estructuras, sino en todo el escenario donde reposan 
las calderas es la velocidad pico de partícula, la cual fue ampliamente superada durante el ensayo con la detonación controlada del modelo a escala de la caldera.

Se puede concluir que durante el análisis general hecho a todo lo que rodea los equipos de producción de vapor y el ensayo con detonación controlada, las vibraciones se mueven de manera vertical a mayor velocidad, por consiguiente, la afectación no solo de toda la vecindad, sino también del espacio aéreo sería muy alta y para este caso muy álgida en el sentido de la cercanía con el aeropuerto internacional el Dorado. Se logra estimar en condiciones normales de operación (27000 libras de agua) la cantidad de energía como onda de choque que entrega la explosión de la caldera de 700 hp a una distancia menor de 0,5 metros; siendo un valor 1.81 veces la presión de detonación del ANFO.

De igual modo, se puede predecir teóricamente la energía de choque de la caldera (presión de detonación ANFO) en condiciones extremas de operación. Es decir, a un volumen de 40.497,28 litros, como gas vapor de agua. En dado caso de una novedad en la embotelladora, se podría decir que difícilmente las tres calderas llegarían a explotar al mismo tiempo, ya que si una explota afectaría a la más cercana, pero por cuestiones de golpe, metal contra metal y no bajo un efecto domino, por consiguiente, la caldera que reciba el golpe físico o de presión de los gases se fracturaría permitiendo la fuga del vapor de forma más focalizada en un punto específico, mas no se fragmentaria. Es importante indicar que existen riesgos de conflagración al momento de la explosión de cualquiera de las calderas por la cercanía con la bodega de azucares y jarabes terminados, los cuales pueden llegar a arder luego de una catálisis generada por la presión de los gases y los resultados de la explosión convirtiéndola en comburente. No se puede recomendar ningún tipo de material que permita mitigar la onda aérea producto de la explosión de la caldera, no hay como realizar una adecuación a la infraestructura existente, no es viable por su localización dentro de la fábrica y por el tipo de estructura donde se encuentran las calderas, alguna intervención a las instalaciones existente generaría una inversión muy alta y realmente seria poco eficaz al momento de cualquier siniestro.

Finalmente, el ideal y como conclusión importante de este trabajo, es que la embotelladora de Coca Cola Femsa mantenga una actualización permanente de la tecnología de los equipos de producción de vapor que día a día se mejoran y están en el mercado respecto al tema de la seguridad industrial a la comunidad científica en el área investigada.

\section{Referencias}

[1] Ejército Nacional, Manual de conocimiento y empleo de explosivos, Imprenta Ejército, Bogotá D.C, (2007).

[2] Indumil, Catálogo de Productos, Características técnicas ANFO agente de voladura. Industria Militar de Colombia, Bogotá D.C, (2019).

[3] ISEE International society of explosives engineers, Manual Del Especialista En Voladuras, Estados Unidos: Sociedad Internacional de Ingenieros de Explosivos (2008).

[4] Manual práctico de voladura EXSA, 2013

[5] H. Montiel, J. Casal, E. Planas y J. A. Vílchez, Análisis del riesgo en instalaciones industriales, (1999).

[6] Ritcher, MSK, Mercalli. Escalas sísmicas. Firestation, Sisrisk, https://firestation.wordpress.com/2009/10/30/escalas-sismicasritcher-msk-mercalli/

[7] Powermaster Wet Back. Ficha de especificaciones técnicas calderas

[8] López Jimeno. Manual de perforación y voladura de roca. Instituto Geominero de España. https://es.scribd.com/document/209393479/ManuaL-dePerforacion-y-Voladura-de-Rocas-LOPEZ-JIMENO

[9] DIN Deutsches Institut für Normung (Instituto de Normalización Alemana), DIN 4150:2000. 